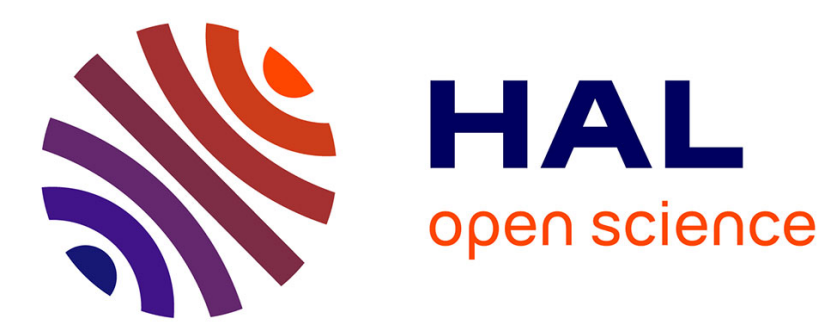

\title{
Surface Electric Field Model for the Beam-Tilted-Foil Interaction
}

\author{
M. Lombardi
}

\section{To cite this version:}

M. Lombardi. Surface Electric Field Model for the Beam-Tilted-Foil Interaction. Physical Review Letters, 1975, 35, pp.1172-1175. 10.1103/PHYSREVLETT.35.1172 . hal-00974328

\section{HAL Id: hal-00974328 \\ https://hal.science/hal-00974328}

Submitted on 6 Apr 2014

HAL is a multi-disciplinary open access archive for the deposit and dissemination of scientific research documents, whether they are published or not. The documents may come from teaching and research institutions in France or abroad, or from public or private research centers.
L'archive ouverte pluridisciplinaire HAL, est destinée au dépôt et à la diffusion de documents scientifiques de niveau recherche, publiés ou non, émanant des établissements d'enseignement et de recherche français ou étrangers, des laboratoires publics ou privés. 
(1973) .

${ }^{2}$ V. G. Lind et al., Phys. Rev. Lett. 32, 479 (1974).

${ }^{3} \mathrm{D}$. Ashery et al., Phys. Rev. Lett. $\underline{32}, 943$ (1974).

${ }^{4}$ The authors of Ref. 2 have recently uncovered an error which, when corrected, lowers their calcium results by a factor of 2.4. Only their calcium data are affected.

${ }^{5}$ R. E. Segel et al., to be published.

${ }^{6}$ H. E. Jackson et al., Phys. Rev. Lett. 35, 641 (1975).

${ }^{7}$ C. E. Wiegand and G. L. Godfrey, Phys. Rev. A $\underline{9}$, 2282 (1974).

${ }^{8}$ P. D. Barnes et al., Phys. Rev. Lett. $\underline{29}, 230$ (1972).

\title{
Surface Electric Field Model for the Beam-Tilted-Foil Interaction
}

\author{
M. Lombardi \\ Laboratoire de Spectrométrie Physique, Université Scientifique et Médicale, 38041 Grenoble-Cedex, France
}

(Received 28 July 1975)

\begin{abstract}
It is shown that the experiments on the polarization of the light emitted after beam-
\end{abstract}
tilted-foil interaction are compatible with a surface electric field model.

Following the theory of Macek, the experimental work of Andrä ${ }^{1}$ has shown that the atoms or ions which are produced in excited states by passing an accelerated beam of ions through a thin carbon foil normal to the beam are aligned; i.e., they emit light partially linearly polarized. More recently, after a theoretical suggestion of Fano and $\mathrm{Macek}^{2}$ and of Ellis, ${ }^{3}$ Berry, Curtis, Ellis, and Schectman ${ }^{4}$ have shown experimentally that when the foil is tilted relative to the beam, an orientation of the excited levels appears in the direction $O x$ perpendicular both to the beam direction $\mathrm{Oz}$ and the foil normal $O z^{\prime}$. This orientation leads to the emission of partially circularly polarized light. Several authors ${ }^{5-7}$ have suggested that the orientation could be caused by the action on the excited state emerging from the foil of an electric field at the final surface of the foil. They have pointed out the similarity of such a mechanism with a previous observation of Giroud and Lombardi. ${ }^{8,9}$ In this last experiment, an electric field of a few hundred volts per centimeter was applied to a set of atoms excited and aligned by electron bombardment. It was then shown that an orientation perpendicular both to the electric field and to the electron beam appeared whenever the beam and the field were neither parallel nor perpendicular and when the phase $\varphi=\tau \Delta \boldsymbol{E} / \hbar$ was of the order of unity ( $\Delta E$ is the Stark splitting, $\tau$ $=30 \mathrm{nsec}$ is the lifetime of the level). If $\varphi \ll 1$, the field has no time to act during the lifetime; if $\varphi \gg 1$, the orientation oscillates rapidly and is averaged out.

$\mathrm{Eck}^{5}$ has made an explicit calculation of such an effect under very restrictive assumptions. He has calculated the effect of a second-order Stark Hamiltonian (or, equivalently, of a first-order electric gradient interaction) upon a $J=1$ level aligned along the beam in the bulk of the foil. He showed first that the maximum of orientation should occur when the tilt angle $\alpha$ of the foil is $45^{\circ}$, second that the fractional polarization percentage $f_{p}$ (with the notations of Ref. 4) is constant when one tilts the foil. These two predictions have been shown to be in contradiction with experiment by Berry, Curtis, and Schectman. ${ }^{10}$ The main purpose of this Comment is to demonstrate that this does not rule out the interpretation of the phenomenon by surface electric field, but only the too restrictive assumptions of Eck. ${ }^{5}$

In the tilted-foil configuration, one has first to account for the existence of a surface electric field. As suggested by Eck, ${ }^{5}$ it may be due to the image charge of the ion in the foil, but in that case one can hardly explain the similarity observed between the results in neutral He and various ionic species. Another possible field source is the direct electrostatic interaction of the incoming ion with the last layer of atoms in the foil. That possibility is not ruled out by surface corrugations either at the atomic $(1 \AA)$ or microscopic $(1 \mu \mathrm{m})$ level. Indeed, even if the electric-field direction is distributed evenly in the forward halfspace of the foil, the $\sin \alpha$ and $\cos \alpha$ terms in the formulas below are reduced only by a factor of 2 . If one uses a more realistic, somewhat forward peaked, distribution of the electric fields, the other terms with higher multiples of $\alpha$ are also preserved, only somewhat reduced. However, in the hypothesis of a last-layer interaction, the 
electric field involved is probably only an effective electric field which takes into account the more complex interaction of ion and foil. The optical electron may come from the bulk with the ion. In this case it probably undergoes at the surface a transition from a low-lying orbital to the final one. Indeed, the excited states involved are usually much too big ( $\simeq 4.5 \AA$ for the $n=3{ }^{4} \mathrm{He} \mathrm{lev-}$ el studied in Ref. 4) to pass through the foil lattice. ${ }^{11}$ The optical electron may alternatively be picked up at the surface. In either case, one has to study the transition from a bound (first case) or unbound (second case) state of the ion or atom under the action of an effective electric field which represents the action of the foil surface. The crucial point, from the point of view of the orientation effect, is that in either case the phase $\varphi$ defined as above is of the order of unity if one supposes $\tau \simeq 10^{-16} \mathrm{sec}\left[(1 \AA) /\left(10^{8} \mathrm{~cm} / \mathrm{sec}\right)\right]$ and $E \simeq 1 \mathrm{eV}$.

Let us consider first the experimental evidence for a maximum of the orientation signal at an angle much higher than $45^{\circ}$. This fact, established with angles of up to $60^{\circ}$ in the $2 s{ }^{1} S-3 p{ }^{1} P$ 5016line of ${ }^{4} \mathrm{He}$ and in the $3 s^{\prime}{ }^{1} D-3 p^{\prime 1} F$ line of Ne III in Ref. 10, has been further confirmed with an angle of $80^{\circ}$ by Andrä. ${ }^{12}$ In order to interpret these results, we shall mention three effects which may give peaking at angles other than $45^{\circ}$. The first two of them are certainly not valid in the 5016- $\AA$ He I line, but they must be kept in mind for more general cases. Let us point out first that the phase $\varphi$ varies with $\alpha$ as $1 / v \cos \alpha .^{5}$ This corresponds to the fact that the rotation of the foil not only tilts the electric field with respect to the beam axis, but also increases the interaction time. This could be a huge effect for sufficiently small $\varphi$ 's and would then lead to an orientation signal peaked nearly at $90^{\circ}$. Such an interpretation is however ruled out in the case of the 5016- $\AA$ He I line. Indeed, the experimental results gathered so far indicate that upon increase of the interaction time, i.e., decrease of the incoming-beam velocity, the orientation signal is not increased but rather slightly decreased. ${ }^{6}$

The second effect which may give maxima at angles other than $45^{\circ}$ is the fact that for $J \geqslant 2$, orientation can arise through coupling with even moments of order $\geqslant 4$ of the levels excited in the bulk. Using the tensor calculations of $\mathrm{Fano}^{13}$ as in Refs. 8 or 9 , one can show that the orientation (proportional to $\rho_{1}^{[1]}$ ) induced from an even multipole of order $k$ in the initial state depends upon $\alpha$ as the rotation matrix element $R_{10}{ }^{k} *(0, \alpha, 0)$ $\propto Y_{1}^{k}(\alpha, 0)$. For $k=4, Y_{1}^{4}(\alpha, 0)$ has a maximum positive at $\simeq 25^{\circ}$ and a minimum negative at $\simeq 69^{\circ}$. Again this explanation is certainly not correct in the 5016- $\AA$ He I line for which $J=1$.

Let us consider finally a third mechanism which seems to provide a satisfactory explanation of all the experimental results. It has been shown ${ }^{9}$ by general symmetry considerations that if one considers only one level in the initial state of the atom (or only levels of the same parity), the orientation must vanish for $\alpha=0$ and $90^{\circ}$, and hence be peaked somewhere in between. More recently it has been shown theoretically ${ }^{14}$ that if one supposes that the initial state of the atom has a nonzero value of $\langle\bar{d}\rangle$, i.e., a coherence between opposite-parity levels, a first-order Stark effect creates an orientation which is nonzero at $90^{\circ}$. This phenomenon is the quantal analog of the torque mechanism suggested by Berry and coworkers. ${ }^{6,10}$ A direct experimental demonstration of $S P$ coherence-induced orientation has been made recently ${ }^{15}$ in a beam-gas experiment on $\mathrm{H}$ and $\mathrm{He}^{+}$.

In order to extend this model to beam-tiltedfoil experiments, one must justify both the existence of nonzero $\langle\bar{d}\rangle$ values and of strong firstorder Stark effects. The nonzero value of $\langle\vec{d}\rangle$ in beam-foil experiments after the exit from the foil (which is our final state here) has been demonstrated experimentally ${ }^{15,16}$ by a method suggested by Eck. ${ }^{17}$ It has however been argued ${ }^{18}$ that coherence between different states cannot exist in our initial state, i.e., at the exit from the bulk, but the predictions of this model developed in Ref. 18 are in contradiction with experiments on $S P$ coherence $^{19}$ and on $S D$ coherence ${ }^{20,21}$ in hydrogenoid atoms. Furthermore a nonzero value of $\langle\bar{d}\rangle$, or equivalently of $\operatorname{Re}\left(\sigma_{s p}\right)$ or of the relative phase of $s$ - and $p$-wave amplitudes, means that the optical electron has not an equal probability of being before or after the ion at the exit from the bulk. This is a very probable consequence of the electron-solid interaction if the optical electron comes from the bulk and is granted when it is picked up at the surface. On the other hand, the need for a first-order Stark effect restricts the usual experiments ${ }^{15,16,19}$ to hydrogenoid atoms, but with crystalline fields which give rise to interaction energies of a few eV, first-order Stark effect is practically always present.

To be more precise, we display the values of the four Stokes components of the light observed in the $O x$ direction, obtained with a calculation analogous to Ref. 5 but with two $s$ and $p$ spinless 
levels coupled by a first-order $-\vec{d} \cdot \vec{E}$ Stark Hamiltonian and separated by a zero-field energy $W_{0}$. In that treatment, spins are ignored as usual since they have no time to interact with the orbit during the relevant time $\left(10^{-16} \mathrm{sec}\right)$. The $s$ level involved can be either a real $s$ level of the ion or merely, in the pickup model, the $s$-wave component of the plane wave which represents the optical electron before the collision:

$$
\begin{aligned}
& I=|b|^{2} \sigma_{s}+\frac{1}{2}\left(|a|^{2}+1\right)\left(\sigma_{0}+\sigma_{1}\right)+\frac{1}{2}\left(|a|^{2}-1\right) \cos 2 \alpha\left(\sigma_{0}-\sigma_{1}\right)+\left[\left(b a^{*}+a b^{*}\right) \sigma_{d}+i\left(b a^{*}-a b^{*}\right) \sigma_{p}\right] \cos \alpha ; \\
& M=|b|^{2} \cos 2 \alpha \sigma_{s}+\frac{1}{2}\left(|a|^{2}-1\right) \cos 2 \alpha\left(\sigma_{0}+\sigma_{1}\right)+\left[\operatorname{Re}(a)+\frac{1}{2}|a-1|^{2} \cos ^{2} 2 \alpha\right]\left(\sigma_{0}-\sigma_{1}\right) \\
& +\cos \alpha\left(\left\{\left[b\left(a^{*}-1\right)+b^{*}(a-1)\right] \cos 2 \alpha+b+b^{*}\right\} \sigma_{d}+i\left\{\left[b\left(a^{*}-1\right)-b^{*}(a-1)\right] \cos 2 \alpha+b-b^{*}\right\} \sigma_{p}\right), \\
& C=|b|^{2} \sin 2 \alpha \sigma_{s}+\frac{1}{2}\left(|a|^{2}-1\right) \sin 2 \alpha\left(\sigma_{0}+\sigma_{1}\right)+\frac{1}{2}|a-1|^{2} \sin 2 \alpha \cos 2 \alpha\left(\sigma_{0}-\sigma_{1}\right) \\
& +\sin \alpha\left(\left\{\left[b\left(a^{*}-1\right)+b^{*}(a-1)\right] \cos 2 \alpha+\left(b a^{*}+b^{*} a\right)\right\} \sigma_{d}\right. \\
& \left.+i\left\{\left[b\left(a^{*}-1\right)-b^{*}(a-1)\right] \cos 2 \alpha+b a^{*}-b^{*} a\right\} \sigma_{p}\right) \\
& S=\frac{1}{2} i\left(\alpha-\alpha^{*}\right) \sin 2 \alpha\left(\sigma_{0}-\sigma_{1}\right)+\left[i\left(b-b^{*}\right) \sigma_{d}-\left(b+b^{*}\right) \sigma_{p}\right] \sin \alpha .
\end{aligned}
$$

Here $I, M, C$, and $S$ are the Stokes parameters. ${ }^{4}$ $a=\langle p, 0|S| p, 0\rangle$ and $b=\langle p, 0|s| s, 0\rangle$ are the two components of the $S$ matrix which describe the transition between the $p, 0$ and $s, 0$ sublevels quantized along the field. In the limiting case in which the interaction energy $-\langle s, 0|d| p, 0\rangle E$ is much smaller than $W_{0}, b \rightarrow 0$ and $a \rightarrow \exp \left[-i \int_{t_{0}}^{\infty} V^{2}(t) /\right.$ $\left.W_{0} d t\right]=\exp (-i \varphi)$, so that one recovers the second-order Stark expressions of Eck. ${ }^{5} \sigma_{s}, \sigma_{0}$, and $\sigma_{1}$ are the density matrix components of $s, 0$, $p, 0$, and $p, 1$ sublevels at the exit of the bulk, quantized along the beam. $\sigma_{d}+i \sigma_{p}=\sigma_{p 0, s 0}$ is the coherence between the $p, 0$ and $s, 0$ sublevels. These formulas display the expected results. The circular polarization $S$ is nonzero for $\alpha=90^{\circ}$. $C$ contains $\sin 2 \alpha$ terms which have no change of sign for $\alpha=45^{\circ}$. $M$ contains $\cos 2 \alpha$ terms which produce the change of sign between 0 and $80^{\circ}$ observed by Andrä. ${ }^{12}$ Finally the fractional percentage polarization $f_{p}$ is no longer constant since there is real creation of orientation from coherence, not merely a transformation between different kinds of polarization. The constancy of $f_{p}$ in Eck's calculation ${ }^{5}$ is only a very peculiar consequence of the constancy of $|\rho|=\sum_{k q}\left|\rho_{q}{ }^{k}\right|^{2}$ demonstrated by Fano. ${ }^{13}$ For a $J=1$ level, $f_{p}$ is proportional to the sum of the terms in $|\rho|$ which vary with the tilt angle. But if $J>1$, optical observation depends only on components with $k \leqslant 2$, and one cannot reconstruct $|\rho|$ from observations in zero field.

In conclusion I should say that a more general calculation, which will be published elsewhere, shows that with such a surface electric field model any $\alpha$ dependence compatible with the change of sign of $C$ and $S$ and invariance of $M$ and $I$ when $\alpha \rightarrow-\alpha$ can be predicted. It is then not possible to give a proof or a disproof of this model, as was supposed in Refs. 5 or 10 , by study of $\alpha$ dependence of observations. One has only to make very detailed studies of the surface interaction phenomenon to compare them with experiments. It would then be interesting to see whether dynamical effects such as distortion of the lattice by the ion are important. They do relax the need of first-order Stark effect to give orientation at a tilt angle of $90^{\circ}$. Indeed, they can give an effective electric field of cylindrical mean symmetry in the bulk and hence an electric field of direction intermediate between beam and foil normal at the surface. But this needs much cleaner experiments since these are surface effects and at a pressure of $10^{-6}$ Torr, commonly used in beamfoil experiments, a monolayer of contaminants is deposited every few minutes.

I am indebted to H. J. Andrä and J. D. Silver for communication of their paper before publication and to M. L. Gaillard for a critical reading and correction of the manuscript.

\footnotetext{
${ }^{1}$ J. Macek, Phys. Rev. A 1 1 , 618 (1970); H. J. Andrä, Phys. Rev. Lett. 25,325 (1970). For further references on alignment beam-foil studies, see e.g., Proceedings of the Third International Conference on Beam Foil Spectroscopy, Nucl. Instrum. Methods 110, (1973).

${ }^{2}$ U. Fano and J. Macek, Rev. Mod. Phys. $\underline{45} 553$ (1973).

${ }^{3}$ D. G. Ellis J. Opt. Soc. Am. $\underline{63}, 1232$ (1973).

${ }^{4}$ H. G. Berry, L. J. Curtis, D. G. Ellis, and R. M. Schectman, Phys. Rev. Lett. $\underline{32}, 751$ (1974).

${ }^{5}$ T. G. Eck, Phys. Rev. Lett. 33, 1055 (1974).

${ }^{6}$ H. G. Berry, S. N. Bhardwaj, L. J. Curtis, and R. M. Schectman, Phys. Lett. 50A, 59 (1974).
} 
${ }^{7}$ D. A. Church, W. Kolbe, M. C. Michel, and T. Hadeishi, Phys. Rev. Lett. 33, 565 (1974).

${ }^{8}$ M. Lombardi and M. Giroud, C. R. Acad. Sci., Ser. B 266,60 (1968).

${ }^{9} \mathrm{M}$. Lombardi, J. Phys. 30,631 (1969).

${ }^{10}$ H. G. Berry, L. J. Curtis, and R. M. Schectman, Phys. Rev. Lett. 34, 509 (1975).

${ }^{11}$ H. G. Berry, D. S. Gemmell, R. E. Holland, J. C. Poizat, J. Remilleux, and J. N. Wotthington, Phys. Lett. 49A, 123 (1974).

${ }^{12} \mathrm{H}$. J. Andrä, to be published.

${ }^{13}$ U. Fano, Phys. Rev. 133, B828 (1964).

${ }^{14}$ M. Lombardi, M. Giroud, and J. Macek, Phys. Rev.
A 11, 1114 (1975).

${ }^{15} \mathrm{M}$. Giroud, M. Lombardi, and A. Zgainsky, to be published.

${ }^{16}$ I. A. Sellin, J. R. Mowat, R. S. Peterson, P. M. Griffon, R. Laubert, and H. H. Haselton, Phys. Rev. Lett. 31, 1335 (1975).

${ }^{17}$ T. G. Eck, Phys. Rev. Lett. 31, 270 (1973).

${ }^{18} \mathrm{~T}$. G. Eck, to be published.

${ }^{19}$ A. Gaupp, H. J. Andrä, and J. Macek, Phys. Rev. Lett. 32, 6 (1974).

${ }^{20}$ D. J. Burns and W. H. Hancock, Phys. Rev. Lett. 27 , 370 (1971).

${ }^{21} \mathrm{~J}$. Desesquelles, private communication.

\title{
Quasimolecular States Responsible for Quantum-Mechanical Phase Interference in Low-Energy $\mathrm{Na}^{+}-\mathrm{Ne}$ Inelastic Collisions
}

\author{
N. H. Tolk, J. C. Tully, C. W. White, J. Kraus, and A. A. Monge \\ Bell Laboratories, Murray Hill, New Jersey 07974 \\ and
}

S. H. Neff

Earlham College, Richmond, Indiana 47347

(Received 31 March 1975)

\begin{abstract}
A simple model is presented which affords an unambiguous and quantitative determination of the quasimolecular states responsible for the striking oscillatory structure in the energy dependence of the polarization components of total cross sections for optical emission due to low-energy $\mathrm{Na}^{+}-\mathrm{Ne}$ collisions. The ${ }^{1} \Pi(\Omega= \pm 1)$ and ${ }^{3} \Pi(\Omega= \pm 2)$ states are identified as the major participants in the quantum-mechanical phase-interference process and not the simple single-electron diabatic states as believed previously.
\end{abstract}

Measurements of striking oscillatory structure in the energy dependence of total cross sections for optical emission due to low-energy (100 eV to $6 \mathrm{keV}$ ) $\mathrm{Na}^{+}-\mathrm{Ne}$ collisions have been reported previously ${ }^{1,2}$ and explained in terms of interfering diabatic molecular-orbital states. ${ }^{1,2}$ In this Comment we propose an alternative model which uniquely relates the final $\mathrm{Ne}(3 p)$ and $\mathrm{Na}(3 p)$ atomic excited states to the specific (NeNa) ${ }^{+}$quasimolecular states participating in the quantummechanical phase-interference phenomena. Conclusions based on the model do not support the previous interpretations. ${ }^{1,2}$

The optical radiation observed in these experiments arises from the excitation of $\mathrm{Ne} I$ and $\mathrm{NaI}$ into $3 p$ electronic states as a result of direct and charge-exchange collision processes, ${ }^{3}$

$$
\mathrm{Na}^{+}+\mathrm{Ne} \rightarrow\left\{\begin{array}{l}
\mathrm{Na}^{+}+\mathrm{Ne} *(3 p), \\
\mathrm{Na} *(3 p)+\mathrm{Ne}^{+}
\end{array}\right.
$$

We have extended the measurements to include the energy dependence of both the perpendicular and the parallel components of the absolute emission cross sections arising from ten Ne I $3 s-3 p$ and two NaI $3 s-3 p$ optical transitions. Examples of the data are given in Fig. 1. In some instances very strong polarization was observed, e.g., Fig。 1(b). Analysis of the results has led to the development of a collision model which accounts quantitatively for the amplitudes of the oscillations in each polarization component of the emission cross sections.

The essential elements of the model are contained in the following three hypotheses:

(1) Oscillations in the energy dependence of the cross sections result from interference between one or more pairs of excited levels which are populated coherently at small internuclear separation $\left(R \sim R_{I}\right)$ and then interact at large separation $\left(R \sim R_{0}\right)$ as schematically represented in Fig. 2. This dual-coupling mechanism first proposed by Rosenthal and Foley ${ }^{4}$ has been estab- 